\title{
Düsseldorfer Kreis
}

\section{Oberste Aufsichtsbehörden für den Datenschutz im nicht- öffentlichen Bereich}

\section{Novellierung des Bundesdatenschutzgesetzes}

Langfassung: Novellierung des Bundesdatenschutzgesetzes in den Bereichen Adressenhandel, Werbung und Datenschutzaudit.

Der Düsseldorfer Kreis begrüßt, dass die Bundesregierung durch eine Novellierung des Bundesdatenschutzgesetzes aus den jüngst bekannt gewordenen Datenschutzverstößen im Bereich der Privatwirtschaft Konsequenzen ziehen möchte.

Die uneingeschränkte Streichung des Listenprivilegs und die Pflicht zur Einholung einer Einwilligung des Betroffenen bei der Übermittlung an Dritte oder bei der Nutzung für Werbezwecke für Dritte sind erforderlich, um das informationelle Selbstbestimmungsrecht der Bürgerinnen und Bürger zu stärken.

Hiervon wird künftig auch die Wirtschaft profitieren. Die geplanten Änderungen ermöglichen es, Werbung zielgerichteter und ohne Streuverluste vorzunehmen und unerwünschte Belästigungen zu vermeiden, so dass das Verbrauchervertrauen in die Datenverarbeitung der Wirtschaft gestärkt wird. Die vorgesehenen Regelungen zur Klarstellung, wann eine wirksame Einwilligung in die Werbenutzung vorliegt, und dass diese nicht mit wichtigen vertraglichen Gegenleistungen gekoppelt werden darf, verbessern die Transparenz und die Freiwilligkeit für den Betroffenen.

Darüber hinaus hat die beim Datenschutzgipfel am 4. September 2008 eingesetzte Länderarbeitsgruppe weitere Vor- schläge zur Verbesserung des Bundesdatenschutzgesetzes unterbreitet, die jedoch bisher nicht berücksichtigt wurden.

Die derzeit geplanten Vorschriften genügen nicht, um künftig im Bereich der privaten Wirtschaft ein ausreichendes $\mathrm{Da}$ tenschutzniveau zu verwirklichen. Hierzu bedarf es zum einen einer angemessenen Ausstattung der Datenschutzaufsichtsbehörden.

Es bedarf zum anderen gemäß den europarechtlichen Vorgaben wirksamer Einwirkungsbefugnisse. Hierzu gehört neben adäquaten Kontroll- und Sanktionsmitteln die Möglichkeit, bei schwerwiegenden Datenschutzverstößen die Erhebung und Verwendung personenbezogener Daten zu untersagen. Auch die Stellung der betrieblichen Datenschutzbeauftragten sollte gestärkt werden.

Die bisherigen Vorschläge des Bundesministeriums des Innern zur Einführung eines Datenschutzaudits sind nicht geeignet, den Datenschutz in der Wirtschaft zu verbessern.

\section{Datenschutzrechtliche Bewertung von digitalen Straßenansichten insbesondere im Internet}

Bei digital erfassten Fotos von Gebäudeund Grundstücksansichten, die über Geokoordinaten eindeutig lokalisiert und damit einer Gebäudeadresse und dem Gebäudeeigentümer sowie den Bewohnern zugeordnet werden können, handelt es sich in der Regel um personenbezogene Daten, deren Erhebung und Verarbeitung nach dem Bundesdatenschutzgesetz zu beurteilen ist.

Die Erhebung, Speicherung und Bereitstellung zum Abruf ist nur zulässig, wenn nicht schutzwürdige Interessen der Betroffenen überwiegen. Bei der Beurteilung schutzwürdiger Interessen ist von Bedeutung, für welche Zwecke die Bilddaten verwendet werden können und an wen diese übermittelt bzw. wie diese veröffentlicht werden.

Die obersten Aufsichtsbehörden sind sich einig, dass die Veröffentlichung von georeferenziert und systematisch bereit gestellten Bilddaten unzulässig ist, wenn hierauf Gesichter, Kraftfahrzeugkennzeichen oder Hausnummern erkennbar sind.

Den betroffenen Bewohnern und Grundstückeigentümern ist zudem die Möglichkeit einzuräumen, der Veröffentlichung der sie betreffenden Bilder zu widersprechen und dadurch die Bereitstellung der Klarbilder zu unterbinden.

Keine schutzwürdigen Interessen bestehen, wenn die Darstellung der Gebäude und Grundstücke so verschleiert bzw. abstrakt erfolgt, dass keine individuellen Eigenschaften mehr erkennbar sind.

- Um die Möglichkeit zum Widerspruch schon vor der Erhebung zu eröffnen, sollte die geplante Datenerhebung mit einem Hinweis auf die Widerspruchsmöglichkeit rechtzeitig vorher bekannt gegeben werden. Die Widerspruchsmöglichkeit muss selbstverständlich auch noch nach der Veröffentlichung bestehen. 